\title{
Jiawei Xianglian Decoction (JWXLD), a Traditional Chinese Medicine (TCM), Alleviates CPT-11-Induced Diarrhea in Mice
}

\author{
Jinhua Lu, ${ }^{1}$ Zechen Lin, ${ }^{2}$ Siyu Huang, ${ }^{3}$ Yiwei Shen, ${ }^{3}$ Jing Jiang, ${ }^{3}$ and Shengyou Lin $\mathbb{I D}^{4}$ \\ ${ }^{1}$ Department of Traditional Chinese Comprehensive Medical Oncology, Hangzhou Cancer Hospital, Hangzhou, China \\ ${ }^{2}$ Oncology Department, Dingqiao Branch of GuangXing Hospital Affiliated to Zhejiang Chinese Medical University, \\ Hangzhou, China \\ ${ }^{3}$ Zhejiang Chinese Medical University, Hangzhou, China \\ ${ }^{4}$ Oncology Department, GuangXing Hospital Affiliated to Zhejiang Chinese Medical University, Hangzhou, China
}

Correspondence should be addressed to Shengyou Lin; linsy0628@126.com

Received 17 August 2019; Revised 19 January 2020; Accepted 12 February 2020; Published 12 March 2020

Academic Editor: Oliver Micke

Copyright (c) 2020 Jinhua Lu et al. This is an open access article distributed under the Creative Commons Attribution License, which permits unrestricted use, distribution, and reproduction in any medium, provided the original work is properly cited.

Irinotecan (CPT-11) is used for therapy of various cancers. However, it has several serious adverse reactions such as diarrhea which is caused by SN-38, the active form of CPT-11. This study aimed to evaluate the effectiveness of Jiawei xianglian decoction (JWXLD), which has been widely used for the treatment of diarrhea in China. In this study, a mouse model with delayed diarrhea was generated by CPT-11. Real-time PCR and enzyme-linked immunosorbent assay (ELISA) were performed to explore intestinal microflora and inflammatory cytokine. Hematoxylin and eosin (H\&E) staining was used to analyze tissue morphology. We found that $0.12,0.23$, and $0.46 \mathrm{~g}$ JWXLD significantly reduced the severity of CPT-11-induced diarrhea. The levels of Lactobacillus (Lacto) and Bifidobacterium (Bifid) were significantly downregulated by CPT-11, and these effects can be reversed by JWXLD treatment. Furthermore, JWXLD was observed to decrease the activity of $\beta$-glucuronidase ( $\beta$-GD). Histopathological data showed that CPT-11 induced severe intestinal mucosal injury, which was characterized as grade 6 , and JWXLD significantly alleviated the injury. In addition, CPT-11 increased the productions of tumor necrosis factor-alpha (TNF- $\alpha$ ), tumor necrosis factor-beta (TNF$\beta$ ), interleukin-6 (IL-6), and interleukin-1 (IL-1), but decreased interleukin-15 (IL-15), interleukin-7 (IL-7), and uridine diphosphate-glucuronosyltransferase 1A1 (UGT1A1). In conclusion, JWXLD can counteract these effects caused by CPT-11 treatment. JWXLD could alleviate CPT-11-induced diarrhea.

\section{Introduction}

Irinotecan (CPT-11), an agent of camptothecin, was firstly isolated from Camptotheca acuminata (family: Nyssaceae) in the early 1960s [1]. CPT-11 exerts a broad spectrum of antitumor activity and has been used for the treatment of many kinds of cancer, including colon cancer [2-4]. However, it can cause serious adverse reactions, such as transient neutropenia and delayed diarrhea. As reported, transient neutropenia occurs in approximately $80 \%$ of patients, and delayed diarrhea occurs in approximately $87 \%$ of patients [5]. Blocking DNA replication and transcription via inhibiting topoisomerase-1 has been demonstrated to be the pharmacological effect of CPT-11 [6]. The metabolic pathway of CPT-11 is complex. At first, CPT- 11 is converted into its active metabolite SN-38 by carboxylesterase (CES) 1 and CES2. SN-38 is subsequently metabolized into SN-38 glucuronide (SN-38G) by uridine diphosphate-glucuronosyltransferase 1A1 (UGT1A1) [7]. The SN-38G can be excreted into the bile and deconjugated to SN38 by $\beta$-glucuronidase $(\beta$-GD) in the intestine, leading to the accumulation of $\mathrm{SN}-38[8,9]$. This $\mathrm{SN}-38$ is considered to induce severe diarrhea [10]. Delayed diarrhea is considered to be one of the dose-limiting factors of CPT-11, so it is urgent to find therapies to control delayed diarrhea induced by CPT- 11 .

To date, various experimental agents and strategies are employed to treat CPT-11-induced delayed diarrhea, including inhibition of SN-38 production, promotion of SN38 adsorption, and suppression of $\beta$-GD activity. For 
example, neomycin [11], cholestyramine/levofloxacin [12], and cefpodoxime [13] are used to alleviate CPT-11-induced delayed diarrhea by inhibiting the production of SN-38. Activated charcoal has been reported to prevent irinotecaninduced diarrhea [14]. Although these strategies can alleviate diarrhea, they cannot completely block delayed diarrhea due to the complex pathogenesis. Traditional Chinese medicines (TCMs) have been used to reduce the side effects of cancerrelated medicines for thousands of years [15]. Jiawei xianglian decoction (JWXLD) is composed of stir-fried Coptis chinensis Franch (Ranunculaceae) (chao huanglian in Chinese), Aucklandia lappa (Compositae) (muxiang in Chinese), Sargentodoxa cuneate Rehder and E.H.Wilson (Lardizabalaceae) (daxueteng in Chinese), Taraxacum mongolicum Hand.-Mazz (Compositae) (pugongying in Chinese), Portulaca oleracea L. (Portulacaceae) (machixian in Chinese), and Euphorbia humifusa Willd (Euphorbioideae) (dijincao in Chinese). Gegen Qinlian decoction, one of the active component is Coptis chinensis, has been shown to alleviate inflammation through suppressing TLR4/NF- $\kappa \mathrm{B}$ signaling in acute ulcerative colitis [16]. Portulaca oleracea [17], Euphorbia humifusa [18], and Aucklandia lappa [19] have been reported to play a role in treating with inflammation. JWXLD is a clinically used drug in China, and it has curative effects in the treatment of diarrhea. However, the exact role of JWXLD in chemotherapy-associated diarrhea remains unclear.

In the current study, we used CPT-11, a frequently used chemotherapeutic drug, to generate diarrhea model and explore how JWXLD can alleviate diarrhea. Our results showed that JWXLD can alleviate diarrhea induced by CPT11.

\section{Methods}

2.1. Materials and Reagents. CPT-11 was purchased from Meilun Biotechnology (Dalian, China). Loperamide was purchased from Xian Janssen Pharmaceutical (Xian, China).

2.2. Preparation of JWXLD. Stir-fried Coptis chinensis, Aucklandia lappa, Sargentodoxa cuneate, Taraxacum mongolicum, Portulaca oleracea, and Euphorbia humifusa were decocted at a rate of $2: 3: 5: 4: 10: 5$ in dry weight. After filtration, the complex was concentrated to $100 \%$, which means that $1 \mathrm{~g}$ raw drugs per milliliter. The detection was kept at $4^{\circ} \mathrm{C}$ in a refrigerator for further use.

2.3. Animals and Treatments. $48 \mathrm{BALB} / \mathrm{C}$ mice (weighing $20 \pm 2 \mathrm{~g}$ ) were obtained from the Shanghai Laboratory Animal Research Center (Shanghai, China). The mice were housed under $12 \mathrm{~h}$ light/dark cycle at $20^{\circ} \mathrm{C}-23^{\circ} \mathrm{C}$ and $40-60 \%$ humidity with free access to food and water. After 1 week of normal diet, the mice were randomly divided into 6 groups (control, CPT-11+loperamide, CPT-11, CPT$11+0.12 \mathrm{~g}$ JWXLD, CPT- $11+0.23 \mathrm{~g}$ JWXLD, and CPT$11+0.46 \mathrm{~g}$ JWXLD) with 8 rats in each group. JWXLD was given to the mice through gavage once a day for 7 days, starting from day 0 to day 7 . All the groups except the control group were given $75 \mathrm{mg} / \mathrm{kg}$ CPT-11 through intraperitoneal injection once a day for 4 days, starting from day 1 to day 5 . The loperamide group was given $0.23 \mathrm{~g}$ loperamide through gavage once a day for 7 days, starting from day 0 to day 7 . The control group was given the same amount of normal saline instead. All experiments were performed in accordance with the National Institutes of Health Guidelines for Animal Research and approved by the Ethics Committee of the Institute of Zhejiang Chinese Medical University.

On the 8th day, mice feces were collected, and all the mice were sacrificed. Ileum and liver of each mouse were cut and kept in a $10 \%$ formalin solution or at $-80^{\circ} \mathrm{C}$ in the refrigerator for further experiments.

2.4. Assessment of Diarrhea. On the third day following the final administration of CPT-11, mice feces were collected to analyze the degree of diarrhea. The severity of diarrhea was scored as follows [20]: 0, normal feces; 1 , soft feces or small black feces; 2 , wet and unformed feces; and 3, watery feces with severe perianal staining of the coat.

2.5. Real-Time PCR. For the analysis of intestinal microflora, $0.2 \mathrm{~g}$ mice normal feces and $1.5 \mathrm{ml}$ phosphate-buffered saline (PBS) were added, mixed for $5 \mathrm{~min}$, and then centrifuged at $1000 \mathrm{rpm}$ for $10 \mathrm{~min}$. The supernatants were treated 3 times as described above. For the last time, the supernatants were centrifuged at $14000 \mathrm{rpm}$ for $10 \mathrm{~min}$, and the sediments were retained. Next, the sediments were washed 4 times with $1 \mathrm{ml}$ PBS. The bacteria were broken with Triton X-100, washed with phenol/chloroform, and deposited with cold ethanol. After being dried at room temperature, bacterial DNA was dissolved with sterile water.

The amplification of intestinal microflora DNA including Lactobacillus (Lacto), Bifidobacterium (Bifid), and Escherichia coli (E. coli) was performed on a TaKaRa PCR amplifier (Dalian, China) using SYBR Green mix assay (TaKaRa, Dalian, China). The procedure is as follows: $95^{\circ} \mathrm{C}$ for $10 \mathrm{~min} ; 95^{\circ} \mathrm{C}$ for $15 \mathrm{sec}$, and $60^{\circ} \mathrm{C}$ for $45 \mathrm{sec}$ with 40 cycles, at last $95^{\circ} \mathrm{C}$ for $15 \mathrm{sec}$ and $60^{\circ} \mathrm{C}$ for $1 \mathrm{~min}$. The DNA copy number was measured by the standard curve.

Lacto

Primer F: $5^{\prime}$-ACGGGAGGCAGCAGTAGGGA- ${ }^{\prime}$ Primer R: 5'-AGCCGTGACTTTCTGGTTGATT- ${ }^{\prime}$

Bifid

Primer F: 5'-GATTCTGGCTCAGGATGAACGC- $3^{\prime}$ Primer R: 5'-CTGATAGGACGCGACCCCAT-3'

E. coli

Primer F: $5^{\prime}$-CATGCCGCGTGTATGAAGAA- $3^{\prime}$

Primer R: $5^{\prime}$-CGGGTAACGTCAATGAGCAAA-3'

For the relative expression level of target genes, the total RNA of liver tissues was extracted using Trizol reagent kit (Invitrogen, Carlsbad, CA, USA) and reverse transcribed to cDNA using cDNA synthesis kit (Promega, Madison, WI, 
USA). The amplification system was used as described above. Relative quantification of the gene expression level was presented using the comparative Ct method $\left(2^{-\Delta \mathrm{Ct}}\right)$ and $\mathrm{GAPDH}$ as the internal reference gene.

\section{UGT1A1}

Primer F: $5^{\prime}$-AGTCTGGCACTCTGCTTTC- $3^{\prime}$ Primer R: $5^{\prime}$-GTGTCACAGCCTCATCTCTTC-3'

\section{GAPDH}

Primer F: $5^{\prime}$-CTGCCCAGAACATCATCC-3' Primer R: $5^{\prime}$-CTCAGATGCCTGCTTCAC-3'

2.6. Analysis of $\beta$-GD. To draw the p-nitrophenol (PNP) standard curve, PNP (Sigma, St. Louis, MO, USA) was diluted into $320,160,80,40,20$, and $0 \mathrm{nmol} / \mathrm{ml}$. Then, $1 \mathrm{ml}$ PBS was added to each tube, and the absorbance at $405 \mathrm{~nm}$ was measured by an auto-microplate reader (Bio-Rad Laboratories, Inc., Hercules, CA, USA). The optical density $(\mathrm{OD})$ value and PNP concentration value were used to draw the standard curve.

For the analysis of $\beta$-GD, $0.1 \mathrm{~g}$ mice feces and $2 \mathrm{ml}$ PBS were added, mixed for $5 \mathrm{~min}$, and then added $0.1 \mathrm{ml}$ nitrophenyl $\beta$-D-glucuronide (Sigma, St. Louis, MO, USA). After incubation for $20 \mathrm{~min}$ at $30^{\circ} \mathrm{C}$, the mix was centrifuged at $3000 \mathrm{rpm}$ for $10 \mathrm{~min}$. The supernatants were measured at $405 \mathrm{~nm}$ using an auto-microplate reader. The activity of $\beta$-GD was calculated by the following equation.

$\beta-\mathrm{GD}(\mathrm{nmol} / \mathrm{g} \times \mathrm{min})=\frac{K(\text { PNP concentration }) \times 2 \mathrm{ml}}{0.1 \mathrm{~g}(\text { the weight of feces }) \times 20 \mathrm{~min}}$,

where $K$ is the concentration of PNP.

2.7. Hematoxylin and Eosin (HહE) Staining. After fixing in a $10 \%$ formalin solution for $48 \mathrm{~h}$, the ileum tissue was embedded with paraffin and cut into sections $(5 \mathrm{~mm}$; Leica RM2125, Germany). Sections were stained with H\&E according to standard methods. Then, a light microscope (Olympus, Tokyo, Japan) was used to collect the images at $\times 200$ magnification. The degree of intestinal mucosal injury was graded according to Chiu et al. [21] as follows: 1, normal mucosal villi; 2 , development of a subepithelial space, usually at the apex of the villi with capillary congestion; 3 , extension of the subepithelial space with moderate lifting of the epithelial layer from the lamina propria; 4, massive epithelial lifting down the sides of the villi and ulceration at the villous tips; 5 , denuded villi with dilated capillaries and increased cellularity of the lamina propria; and 6, degradation and disintegration of the lamina propria, hemorrhage, and ulceration.

2.8. Enzyme-Linked Immunosorbent Assay (ELISA). First, frozen ileum samples were homogenized in iced PBS. ELISA kits (Meso Scale Discovery, Rockville, MD, USA) were used to determine IL-1, IL-6, IL-7, IL-15, TNF- $\alpha$, and TNF- $\beta$ from ileum samples according to the manufacturer's instructions.
2.9. Western Blot. First, frozen liver samples were homogenized in RIPA lysis buffer (Solarbio, Beijing, China) and centrifuged at $12000 \mathrm{rpm}$ for $10 \mathrm{~min}$ at $4^{\circ} \mathrm{C}$. Then, the supernatant was separated by $10-15 \%$ SDS-PAGE gel (the concentration of the gel was decided according to the protein molecular weight), followed by transferring to nitrocellulose membranes (Millipore Corp., Bedford, MA, USA). The membranes were blocked with TBST containing $5 \%$ milk for $1 \mathrm{~h}$ at room temperature and incubated with UGT1A1 (Abcam, 1:700 dilution) and GAPDH (Abcam, 1: 2000 dilution) primary antibody at $4^{\circ} \mathrm{C}$ overnight. Then, the membranes were washed 3 times with TBST and incubated with secondary antibodies for $1 \mathrm{~h}$. After 2 times washing, the brands were developed by an enhanced chemiluminescence (ECL) kit (Millipore, Burlington, MA, USA) and collected with the scanner. GAPDH was used as an internal control.

2.10. Statistical Analysis. All values are showed as mean \pm SD. One-way analysis of variance (ANOVA) was used to analyze data of different groups. $P<0.05$ was considered significant.

\section{Results}

3.1. Effect of JWXLD on the Diarrhea Score in the CPT-11Induced Delayed Diarrhea Model. To generate a CPT-11induced delayed diarrhea mouse model, $75 \mathrm{mg} / \mathrm{kg}$ CPT-11 was injected once a day for 4 days [22]. On the third day following the final administration of CPT-11, mice feces were collected to analyze the diarrhea degree. We found that CPT-11 caused severe diarrhea, and all the three doses of JWXLD significantly reduced the severity of diarrhea induced by CPT-11, and $0.23 \mathrm{~g}$ of JWXLD exhibited the best effect. Loperamide, a frequently used antidiarrheic drug, also prominently alleviated diarrhea (Table 1).

3.2. JWXLD Increased Intestinal Microflora Contents in the CPT-11-Induced Delayed Diarrhea Model. As reported, intestinal microflora plays a role in the intestinal toxicity of irinotecan. E. coli is reported to produce $\beta$-GD [23], Lacto is suggested to inhibit $\beta$-GD [24], and Bifid is reported to have protective properties towards the gut mucosal barrier [25]. In order to understand the role of JWXLD, intestinal microflora including Lacto, Bifid, E. coli and $\beta$-GD were measured. As shown in Figure 1, levels of Lacto and Bifid were significantly decreased after treated with CPT-11 and all the three doses of JWXLD markedly increased these contents compared to CPT11 , and $0.23 \mathrm{~g}$ of JWXLD exhibited the best effect. While the level of E. coli was not changed at all. We also found that the activity of $\beta$-GD was inhibited by JWXLD compared with CPT-11, and $0.23 \mathrm{~g}$ of JWXLD showed the highest inhibition (Figure 1). Loperamide showed the similar effects as JWXLD.

3.3. JWXLD Relieved Pathogenesis in the CPT-11-Induced Delayed Diarrhea Model. The condition of the ileum tissue was analyzed by HE staining. CPT-11 induced the 
TABLE 1: Diarrhea score was analyzed after treatment with different doses of JWXLD in CPT-11-induced delayed diarrhea mouse model.

\begin{tabular}{lcccccc}
\hline & & & \multicolumn{3}{c}{ CPT-11 $(75 \mathrm{mg} / \mathrm{kg})$} \\
\hline Group & Control & Loperamide & 0 & 0.12 & 0.23 & 0.46 \\
\hline Average values of diarrhea scores & 0 & $1.87^{* \#}$ & $2.56^{*}$ & $1.12^{* \#}$ & $0.12^{* \#}$ & $0.82^{* \#}$ \\
\hline
\end{tabular}

Data represent average values of diarrhea scores $(n=8) .{ }^{*}, P<0.05$ compared to control mice; ${ }^{\#}, P<0.05$ compared to the mice treated with CPT-11.

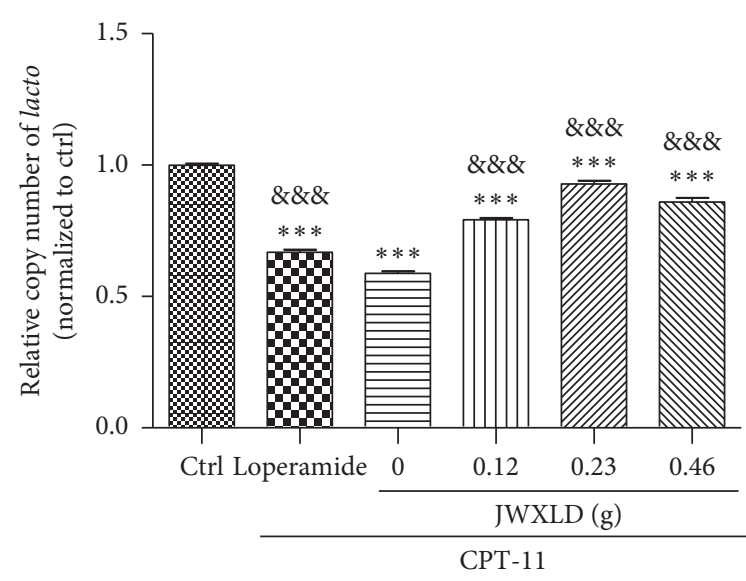

(a)

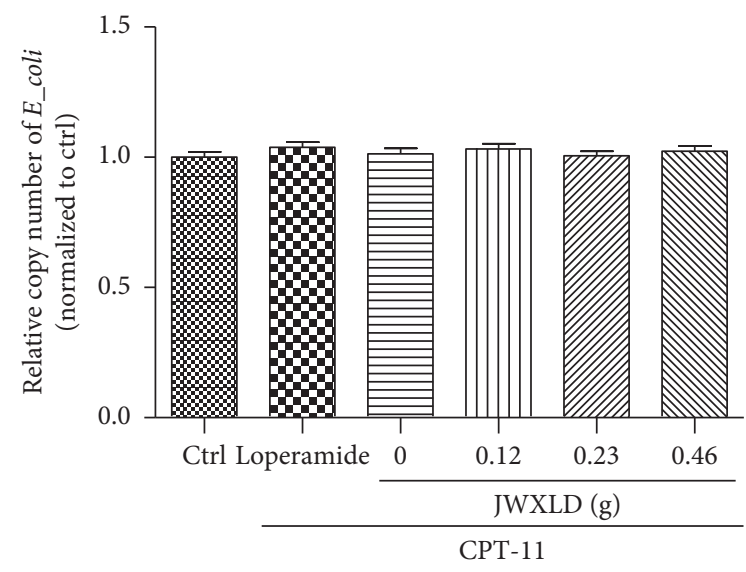

(c)

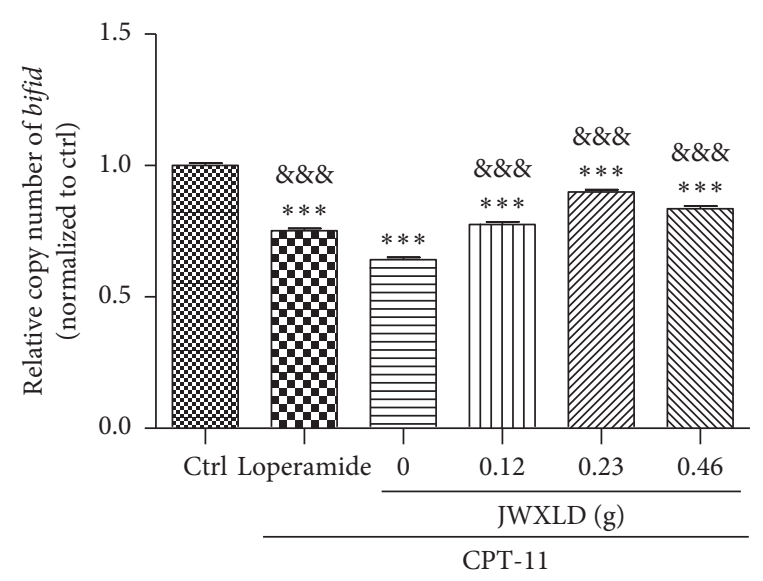

(b)

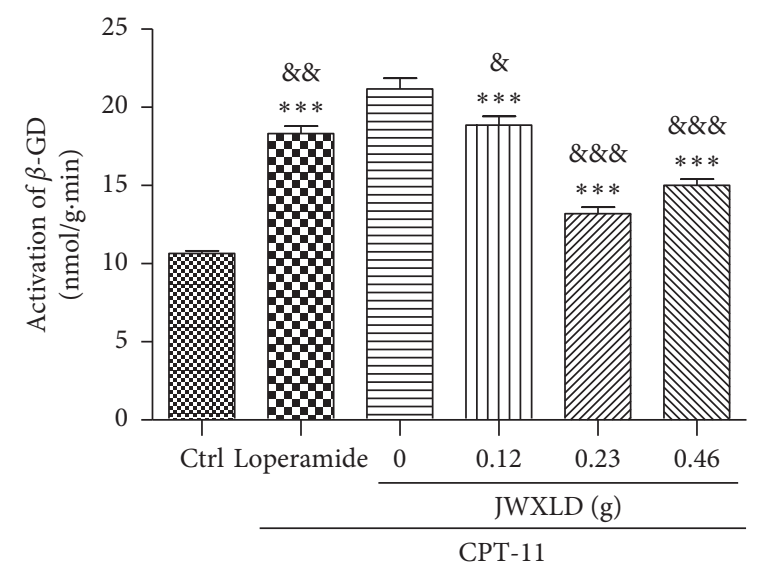

(d)

FIGURE 1: Effect of JWXLD on intestinal microflora in the CPT-11-induced delayed diarrhea model. A, B, C: DNA copy numbers of Lacto, Bifid and E. coli were measured by real-time PCR. D: Activity of $\beta$-GD was measured after treated with CPT-11 and/or different doses of JWXLD. ${ }^{* * *}, P<0.001$ vs control; $\& \& \&, P<0.001$ vs. CPT-11. (a) Lacto. (b) Bifid. (c) E. coli (d). $\beta$-GD.

shortening of the villi, the damage of crypt cells, and infiltration of inflammatory cells into the lamina propria (Figure 2(c)) and characterized intestinal mucosal injury of grade 5.87 (Table 2). JWXLD significantly alleviated the histopathological alterations which were observed in the CPT-11 group (Figure 2(d)-2(f)) (grade 4, 3, 4). Treatment with $0.23 \mathrm{~g}$ of JWXLD increased the villi, reduced the infiltration of inflammatory cells and repaired crypt cells and architecture (Figure 2(e)) (grade 3). In addition, loperamide showed the similar effects as JWXLD (grade 4).

3.4. JWXLD Inhibited the Production of Proinflammatory Cytokines but Promoted Anti-Inflammatory Cytokines in CPT-11-Induced Delayed Diarrhea Model. Pro- and antiinflammatory cytokine expressions have been showed to play a key role in the pathogenesis of diarrhea [22, 26, 27]. As shown in Figure 3(a), CPT-11 induced high production levels of TNF- $\alpha$, TNF- $\beta$, IL- 6 , and IL- 1 of ileum tissues. All the three doses of JWXLD markedly inhibited the production of proinflammatory cytokines induced by CPT-11, and $0.23 \mathrm{~g}$ of JWXLD exhibited the best effect. On the contrary, CPT-11 significantly decreased the ileum tissue level of IL-15 and IL-7, and these inhibitions could be counteracted by JWXLD (Figure 3(b)). Loperamide showed the similar effects as JWXLD.

3.5. JWXLD Overcame the Inhibition of UGT1A1 Induced by CPT-11. As reported [28], UGT1A1 mediates conversion of the active SN-38 to inactive SN-38G and subsequently alleviates delayed diarrhea induced by SN-38. Then, we 


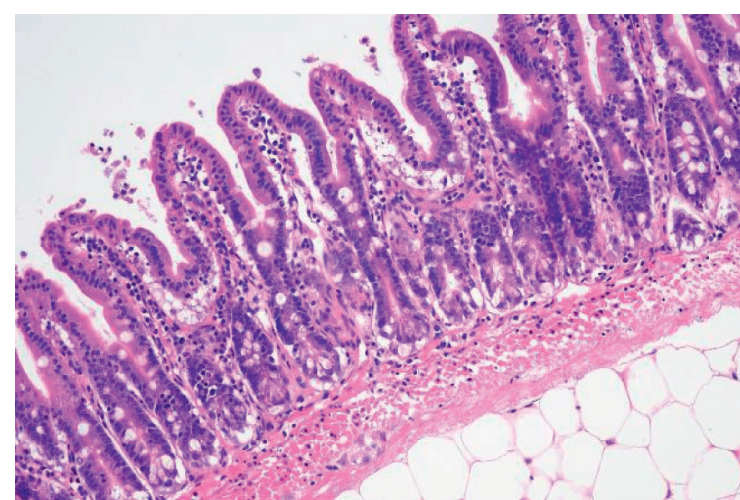

(a)

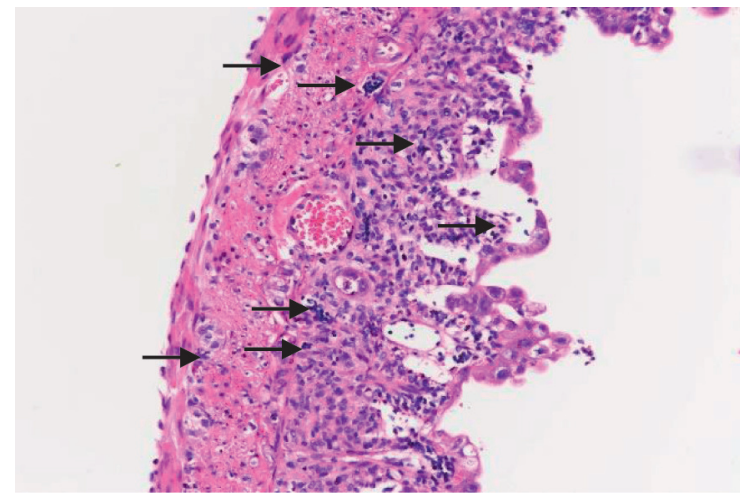

(c)

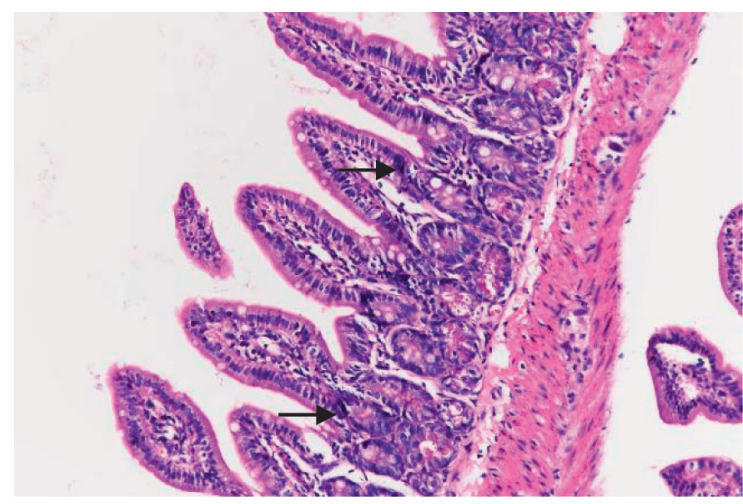

(e)

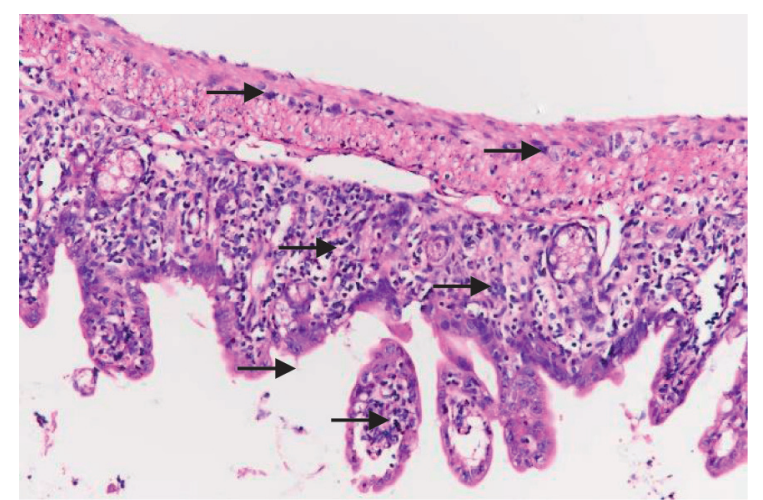

(b)

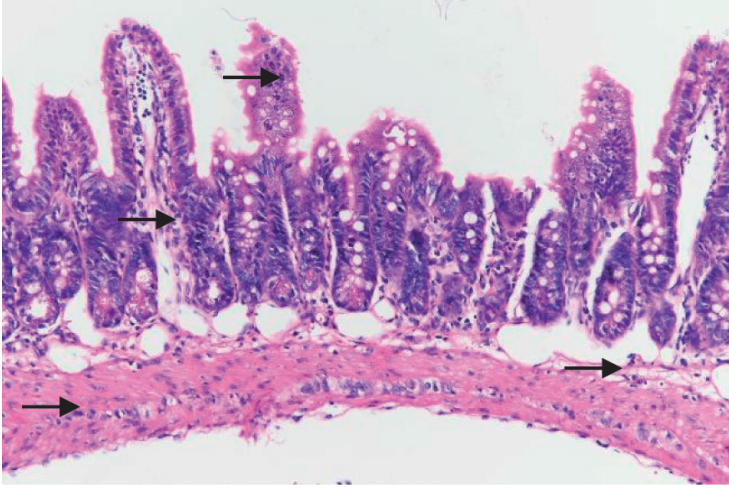

(d)

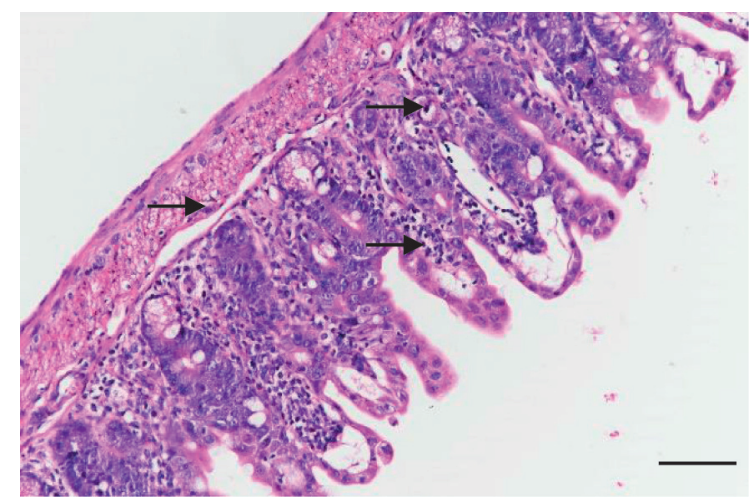

(f)

Figure 2: H\&E staining of ileum tissues. (a) Control group; (b) CPT-11 + loperamide group; (c) CPT-11 group; (d) CPT-11 + $0.12 \mathrm{~g}$ JWXLD group; (e) CPT-11+0.23 g JWXLD group; (f) CPT-11+0.46 g JWXLD group. The black arrows indicate infiltration by inflammatory cells. Scale: $100 \mu \mathrm{m}$.

TABLE 2: Intestinal mucosal injury grade was analyzed after administration of CPT-11 of mice treated with different doses of JWXLD.

\section{CPT-11 $(75 \mathrm{mg} / \mathrm{kg})$}

JWXLD (g)

\begin{tabular}{|c|c|c|c|c|c|c|}
\hline Group & Control & Loperamide & 0 & 0.12 & 0.23 & 0.46 \\
\hline Average intestinal mucosal injury grade & 1.25 & $4.75^{* \#}$ & $5.87 *$ & $4.25^{* \#}$ & $2.75^{* \#}$ & $3.25^{* \#}$ \\
\hline
\end{tabular}

Data represent average values of intestinal mucosal injury grade $(n=8) .{ }^{*} P<0.05$ compared to control mice; ${ }^{\#} P<0.05$ compared to the mice treated with CPT-11. 

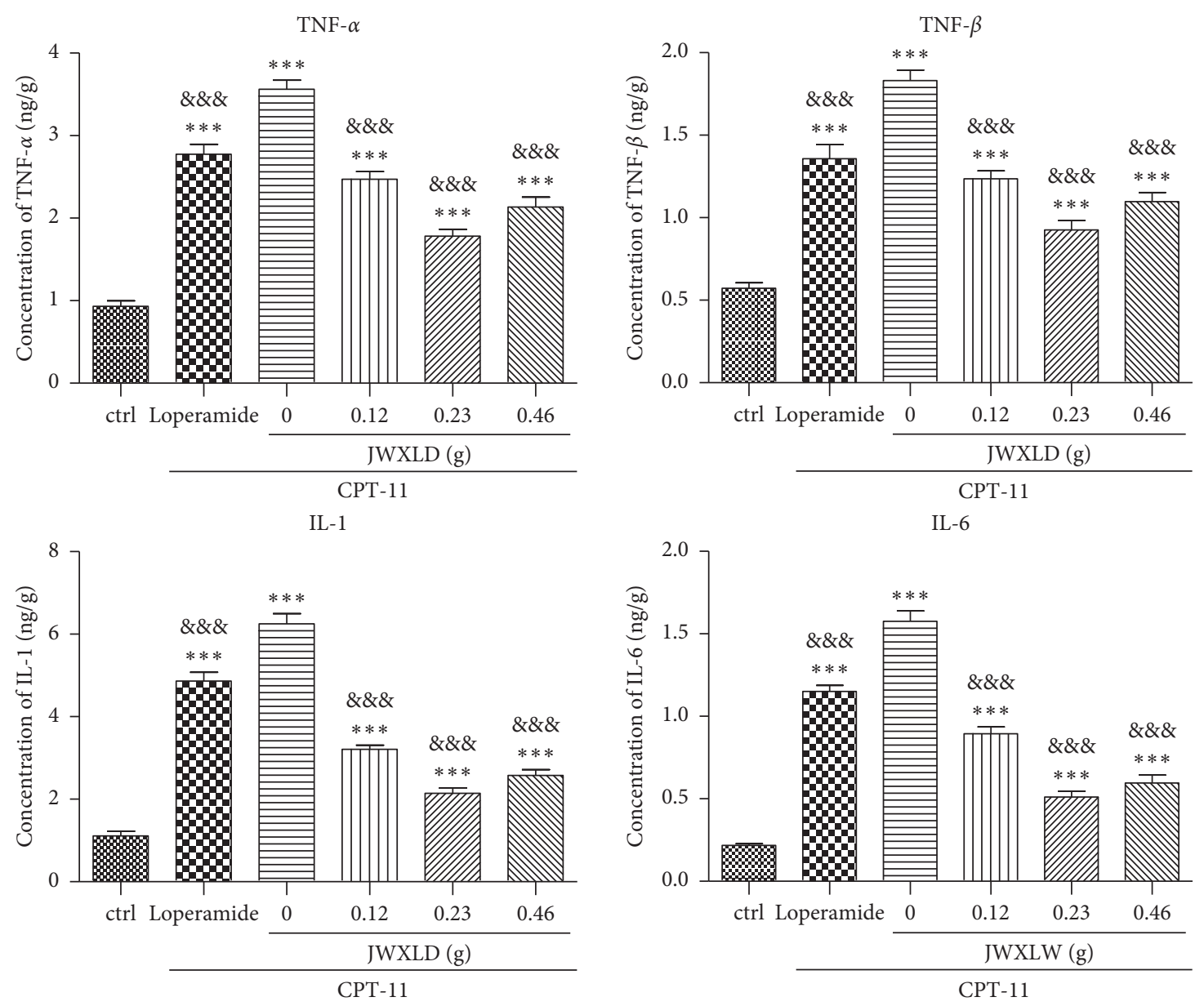

(a)
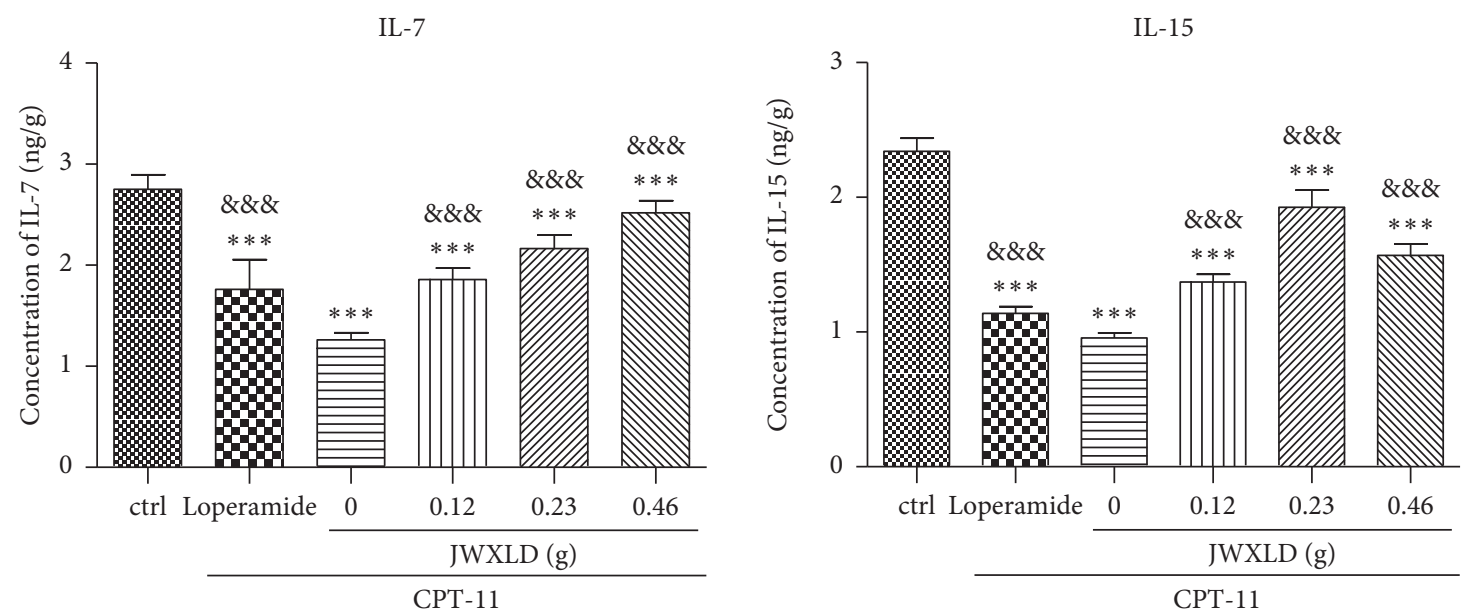

(b)

Figure 3: (a, b) JWXLD inhibited the production of proinflammatory cytokines in the CPT-11-induced delayed diarrhea model. The production levels of TNF- $\alpha$, TNF- $\beta$, IL-6, IL-1, IL-15 and IL-7 were measured using ELISA assay. ${ }^{* * *}, P<0.001$ vs. control; $\& \& \&, P<0.001$ vs. CPT-11.

analyzed the expression level of UGT1A1 after treatment with CPT-11 and/or JWXLD. Interestingly, we observed that CPT11 inhibited the expression of UGT1A1 at mRNA and protein levels, and this inhibition can be partly reversed by JWXLD and loperamide (Figure 4).

\section{Discussion}

As a frequent side effect in patients receiving chemotherapy, chemotherapy-induced diarrhea (CID) was reported as high as $50-80 \%$ in patients treated with 


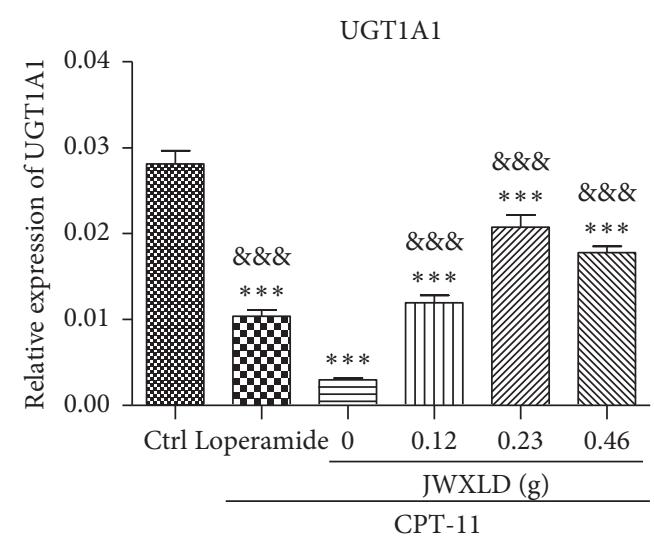

(a)

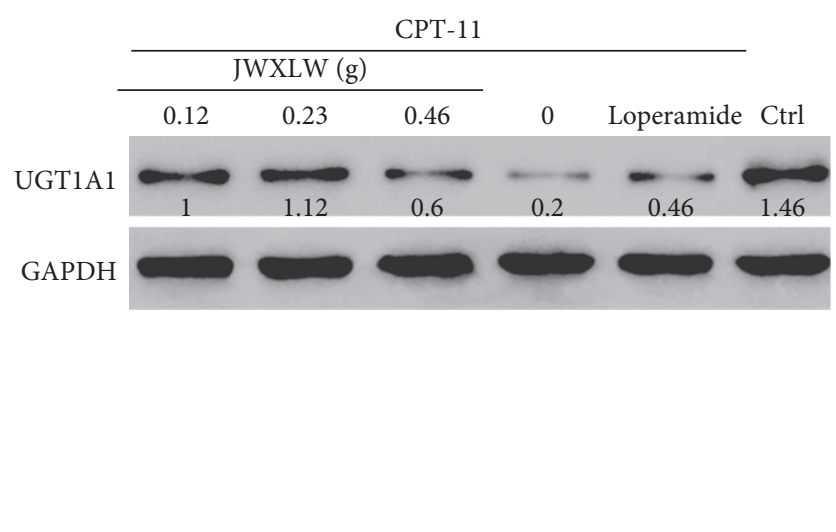

(b)

FIGURE 4: JWXLD reverses the inhibition of UGT1A1 induced by CPT-11. The expression level of liver UGT1A1 was measured by real-time PCR (upper panel) and Western blot (lower panel) $(n=8)$. ${ }^{* * *}, P<0.001$ vs. control; $\& \& \&, P<0.001$ vs. CPT-11.

irinotecan [29]. Germ-free mice showed more resistance to lethal dosage of CPT-11 by 2.5 folds than that of holoxenic mice [30]. The underlying mechanism contributing to CPT-11-induced diarrhea was shown that SN-38G can be hydrolyzed to SN-38 by bacterial $\beta$-GD, thus leading to intestinal toxicity. Thus, inhibiting the activity of $\beta-\mathrm{GD}$ is expected to alleviate diarrhea induced by CPT-11 [29].

Recently, the inhibition of AIM2 by thalidomide, an inflammasome inhibitor, is found to alleviate CPT-11-induced intestinal toxicity without compromising its anticancer efficacy [31]. In addition, one method to protect the intestine from damage induced by reactivation of SN-38 is to inhibit bacterial activity of the intestinal lumen. More severe intestinal histology injury and higher levels of proinflammatory IL- $1 \beta$ and TNF- $\alpha$ cytokines are observed in conventional mice after irinotecan treatment compared with germ-free mice [32]. D-Saccharic acid 1.4-lactone (SAL) was reported by Fittkau et al. which has the effect to reduce irinotecan-induced mucosal damage in rats [33]. Similarly, another group found inhibitor 1 , a specific potent bacterial $\beta$-GD inhibitor, significantly suppressed CPT-11-induced diarrhea and intestinal damage in mice [34]. Unexpectedly, pharmacokinetics of SN38 in plasma which used to determine antitumor efficacy of CPT-11, may be simultaneously influenced by such inhibition. Gupta et al. indicated that SN-38 was absorbed from the intestine as the dominant peak of SN-38 in serum was observed after intravenous infusion of CPT-11 in patients [35]. These findings indicate the enterohepatic recirculation of SN38. Based on those studies, inhibition of bacterial $\beta$-GD activity could relieve the antitumor efficacy of CPT-11 by increasing the level of SN-38G and reducing the reabsorption level of SN-38 and in the intestine.

In this study, we explored that JWXLD, a traditional Chinese herbal decoction, could alleviate diarrhea induced by CPT-11. Firstly, $75 \mathrm{mg} / \mathrm{kg}$ CPT-11 was treated once a day for 4 days, and severe diarrhea was developed by the third day following the final administration. These results indicated that CPT-11 can cause delayed diarrhea (grade 2.56) [36, 37], and different doses of JWXLD can alleviate diarrhea (Table 1).
However, $0.23 \mathrm{~g}$ of JWXLD rather than $0.46 \mathrm{~g}$ of JWXLD exhibited the best effect (grade 0.12 ), which might be caused by the following reason. A traditional Chinese herbal decoction usually has a dose-dependent curve in a range of doses, and $0.46 \mathrm{~g}$ of JWXLD may be above this range so that it does not match the dose-dependent curve. Next, we found that CPT-11 decreased levels of Lacto and Bifid, but failed to regulate the level of E. coli (Figure 1). Lacto has been found to be inhibited after treatment with CPT-11 [38] and Bifid longum (B. longum) has been found to prevent small intestinal mucositis induced by CPT-11 [39]. In this study, JWXLD was observed to increase the levels of Lacto and Bifid compared with CPT-11. In agreement, we found that JWXLD treatment significantly inhibited $\beta$-GD upon treatment with higher dosage (Figure 1). Hepatic UGT1A1 can glucuronidate SN-38 to inactivated SN38G [15]. In this study, we observed increase of UGT1A1 with treatment of JWXLD in Figure 4. Collectively, JWXLD treatment inhibited bacterial $\beta$-GD and increased UGT1A1 and finally prevented diarrhea induced by CPT-11 (Figure 2). In consistent, bacterial contents of Lacto and Bifid were increased as well (Figure 1). Furthermore, proinflammatory (TNF- $\alpha$, TNF- $\beta$, IL-1, and IL-6) were suppressed supporting its protective effects of JWXLD in CPT-11-induced diarrhea (Figure 3). CPT-11 has been reported to significantly increase the level of inflammatory cytokines such as TNF- $\alpha$, IL-1, and IL-6 in the rat colon [40], which is consistent with our findings. However, how JWXLD alleviates inflammation is still not clear. Fang et al. reported CPT-11-induced elevation of bile acids which potentiates suppression of IL-10 expression [41]. Kon et al. revealed CPT-11-induced delayed diarrhea develops via reduced aquaporin-3 expression in the colon [40]. Li et al. found CPT-11 activates NLRP3 inflammasome through JNK and NF- $\kappa$ B signaling pathway $[42,43]$. For clinical application purpose, what are the effects of JWXLD on antitumor efficacy of CPT-11 need be explored more in future.

\section{Conclusion}

In summary, we showed JWXLD, an important component of TCMs, prevents CPT-11-induced diarrhea. 


\section{Data Availability}

The data used to support the findings of this study are available from the corresponding author upon request.

\section{Conflicts of Interest}

The authors declare that they have no conflicts of interest.

\section{Acknowledgments}

This work was supported by the Science Technology Department of Zhejiang Province (2018C03025).

\section{References}

[1] R. H. Mathijssen, R. J. van Alphen, J. Verweij et al., "Clinical pharmacokinetics and metabolism of irinotecan (CPT-11)," Clinical Cancer Research: An Official Journal of the American Association for Cancer Research, vol. 7, no. 7, pp. 2182-2194, 2001.

[2] X. Xiao, S. Xia, M. Zou et al., "The relationship between UGT1A1 gene polymorphism and irinotecan effect on extensive-stage small-cell lung cancer," OncoTargets and Ther$a p y$, vol. 8, pp. 3575-3583, 2015.

[3] M. Kim, B. Keam, T.-M. Kim et al., "Phase II study of irinotecan and cisplatin combination chemotherapy in metastatic, unresectable esophageal cancer," Cancer Research and Treatment, vol. 49, no. 2, pp. 416-422, 2017.

[4] C. Xu, X. Tang, Y. Qu, S. Keyoumu, N. Zhou, and Y. Tang, "UGT1A1 gene polymorphism is associated with toxicity and clinical efficacy of irinotecan-based chemotherapy in patients with advanced colorectal cancer," Cancer Chemotherapy and Pharmacology, vol. 78, no. 1, pp. 119-130, 2016.

[5] H. Bleiberg and E. Cvitkovic, "Characterisation and clinical management of CPT-11 (irinotecan)-induced adverse events: the European perspective," European Journal of Cancer, vol. 32, no. 3, pp. S18-S23, 1996.

[6] S. Marsh and J. M. Hoskins, "Irinotecan pharmacogenomics," Pharmacogenomics, vol. 11, no. 7, pp. 1003-1010, 2010.

[7] L. Iyer, C. D. King, P. F. Whitington et al., "Genetic predisposition to the metabolism of irinotecan (CPT-11). Role of uridine diphosphate glucuronosyltransferase isoform $1 \mathrm{~A} 1$ in the glucuronidation of its active metabolite (SN-38) in human liver microsomes," Journal of Clinical Investigation, vol. 101, no. 4, pp. 847-854, 1998.

[8] K. Toshimoto, A. Tomaru, M. Hosokawa, and Y. Sugiyama, "Virtual clinical studies to examine the probability distribution of the auc at target tissues using physiologically-based pharmacokinetic modeling: application to analyses of the effect of genetic polymorphism of enzymes and transporters on irinotecan induced side effects," Pharmaceutical Research, vol. 34, no. 8, pp. 1584-1600, 2017.

[9] K. Takasuna, T. Hagiwara, M. Hirohashi et al., "Involvement of beta-glucuronidase in intestinal microflora in the intestinal toxicity of the antitumor camptothecin derivative irinotecan hydrochloride (CPT-11) in rats," Cancer Research, vol. 56, no. 16 , pp. 3752-3757, 1996.

[10] U. Swami, S. Goel, and S. Mani, "Therapeutic targeting of CPT-11 induced diarrhea: a case for prophylaxis," Current Drug Targets, vol. 14, no. 7, pp. 777-797, 2013.

[11] F. A. de Jong, D. F. S. Kehrer, R. H. J. Mathijssen et al., "Prophylaxis of irinotecan-induced diarrhea with neomycin and potential role for $\mathrm{UGT} 1 \mathrm{~A} 1 * 28$ genotype screening: a double-blind, randomized, placebo-controlled study," The Oncologist, vol. 11, no. 8, pp. 944-954, 2006.

[12] D. Flieger, C. Klassert, S. Hainke et al., "Phase II clinical trial for prevention of delayed diarrhea with cholestyramine/levofloxacin in the second-line treatment with irinotecan biweekly in patients with metastatic colorectal carcinoma," Oncology, vol. 72, no. 1-2, pp. 10-16, 2007.

[13] L. M. McGregor, C. F. Stewart, K. R. Crews et al., "Dose escalation of intravenous irinotecan using oral cefpodoxime: a phase I study in pediatric patients with refractory solid tumors," Pediatric Blood \& Cancer, vol. 58, no. 3, pp. 372-379, 2012.

[14] G.-C. Sergio, G.-M. Félix, and J. V. Luis, "Activated charcoal to prevent irinotecan-induced diarrhea in children," Pediatric Blood \& Cancer, vol. 51, no. 1, pp. 49-52, 2008.

[15] H. Y. Guan, P. F. Li, X. M. Wang et al., "Shengjiang xiexin decoction alters pharmacokinetics of irinotecan by regulating metabolic enzymes and transporters: a multi-target therapy for alleviating the gastrointestinal toxicity," Frontiers in Pharmacology, vol. 8, p. 769, 2017.

[16] R. Li, Y. Chen, M. Shi et al., "Gegen Qinlian decoction alleviates experimental colitis via suppressing TLR4/NF- $\kappa \mathrm{B}$ signaling and enhancing antioxidant effect," Phytomedicine, vol. 23, no. 10, pp. 1012-1020, 2016.

[17] Y. X. Zhou, H. L. Xin, K. Rahman et al., "Portulaca oleracea L.: a review of phytochemistry and pharmacological effects," BioMed Research International, vol. 2015, Article ID 925631, 11 pages, 2015.

[18] B. T. T. Luyen, B. H. Tai, N. P. Thao et al., "Anti-inflammatory components of Euphorbia humifusa Willd," Bioorganic \& Medicinal Chemistry Letters, vol. 24, no. 8, pp. 1895-1900, 2014.

[19] J.-H. Woo, J.-H. Ahn, D. S. Jang, and J.-H. Choi, "Effect of dehydrocostus lactone isolated from the roots of aucklandia lappa on the apoptosis of endometriotic cells and the alternative activation of endometriosis-associated macrophages," The American Journal of Chinese Medicine, vol. 47, no. 6, pp. 1289-1305, 2019.

[20] A. Kurita, S. Kado, T. Matsumoto et al., "Streptomycin alleviates irinotecan-induced delayed-onset diarrhea in rats by a mechanism other than inhibition of $\beta$-glucuronidase activity in intestinal lumen," Cancer Chemotherapy and Pharmacology, vol. 67, no. 1, pp. 201-213, 2011.

[21] C.-J. Chiu, A. H. McArdle, R. Brown, H. J. Scott, and F. N. Gurd, "Intestinal mucosal lesion in low-flow states," Archives of Surgery, vol. 101, no. 4, pp. 478-483, 1970.

[22] M. L. P. Melo, G. A. C. Brito, R. C. Soares et al., "Role of cytokines (TNF- $\alpha$, IL- $1 \beta$ and KC) in the pathogenesis of CPT11-induced intestinal mucositis in mice: effect of pentoxifylline and thalidomide," Cancer Chemotherapy and Pharmacology, vol. 61, no. 5, pp. 775-784, 2008.

[23] A. M. Stringer, R. J. Gibson, R. M. Logan, J. M. Bowen, A. S. Yeoh, and D. M. Keefe, "Faecal microflora and $\beta$-glucuronidase expression are altered in an irinotecan-induced diarrhea model in rats," Cancer Biology \& Therapy, vol. 7, no. 12, pp. 1919-1925, 2008.

[24] O. Sreekumar and A. Hosono, "Immediate effect of Lactobacillus acidophilus on the intestinal flora and fecal enzymes of rats and the in vitro inhibition of Escherichia coli in coculture," Journal of Dairy Science, vol. 83, no. 5, pp. 931-939, 2000.

[25] B. Kleessen and M. Blaut, "Modulation of gut mucosal biofilms," British Journal of Nutrition, vol. 93, no. S1, pp. S35-S40, 2005.

[26] Y. Wu, D. Wang, X. Yang, C. Fu, L. Zou, and J. Zhang, "Traditional Chinese medicine Gegen Qinlian decoction 
ameliorates irinotecan chemotherapy-induced gut toxicity in mice," Biomedicine \& Pharmacotherapy, vol. 109, pp. 22522261, 2019.

[27] C. Takasu, W. G. Yismaw, N. Kurita et al., "TU-100 exerts a protective effect against bacterial translocation by maintaining the tight junction," Surgery Today, vol. 47, no. 10, pp. 1287-1294, 2017.

[28] K. Inoue, M. Sonobe, Y. Kawamura et al., "Polymorphisms of the UDP-glucuronosyl transferase 1A genes are associated with adverse events in cancer patients receiving irinotecanbased chemotherapy," The Tohoku Journal of Experimental Medicine, vol. 229, no. 2, pp. 107-114, 2013.

[29] K.-W. Cheng, C.-H. Tseng, C.-C. Tzeng et al., "Pharmacological inhibition of bacterial $\beta$-glucuronidase prevents irinotecan-induced diarrhea without impairing its antitumor efficacy in vivo," Pharmacological Research, vol. 139, pp. 4149, 2019.

[30] G. Brandi, J. Dabard, P. Raibaud et al., "Intestinal microflora and digestive toxicity of irinotecan in mice," Clinical Cancer Research, vol. 12, no. 4, pp. 1299-1307, 2006.

[31] Q. Lian, J. Xu, S. Yan et al., "Chemotherapy-induced intestinal inflammatory responses are mediated by exosome secretion of double-strand DNA via AIM2 inflammasome activation," Cell Research, vol. 27, no. 6, pp. 784-800, 2017.

[32] S. H. S. P. Pedroso, A. T. Vieira, R. W. Bastos et al., "Evaluation of mucositis induced by irinotecan after microbial colonization in germ-free mice," Microbiology, vol. 161, no. 10, pp. 1950-1960, 2015.

[33] M. Fittkau, W. Voigt, H. J. Holzhausen, and H. J. Schmoll, "Saccharic acid 1.4-lactone protects against CPT-11-induced mucosa damage in rats," Journal of Cancer Research and Clinical Oncology, vol. 130, no. 7, pp. 388-394, 2004.

[34] B. D. Wallace, H. Wang, K. T. Lane et al., "Alleviating cancer drug toxicity by inhibiting a bacterial enzyme," Science, vol. 330, no. 6005, pp. 831-835, 2010.

[35] E. Gupta, T. M. Lestingi, R. Mick, J. Ramirez, E. E. Vokes, and M. J. Ratain, "Metabolic fate of irinotecan in humans: correlation of glucuronidation with diarrhea," Cancer Research, vol. 54, pp. 3723-3725, 1994.

[36] R. J. Gibson, J. M. Bowen, M. R. Inglis, A. G. Cummins, and D. M. Keefe, "Irinotecan causes severe small intestinal damage, as well as colonic damage, in the rat with implanted breast cancer," Journal of Gastroenterology and Hepatology, vol. 18, no. 9, pp. 1095-1100, 2003.

[37] R. M. Logan, R. J. Gibson, J. M. Bowen, A. M. Stringer, S. T. Sonis, and D. M. K. Keefe, "Characterisation of mucosal changes in the alimentary tract following administration of irinotecan: implications for the pathobiology of mucositis," Cancer Chemotherapy and Pharmacology, vol. 62, no. 1, pp. 33-41, 2008.

[38] W. Lam, Z. Jiang, F. Guan et al., "The number of intestinal bacteria is not critical for the enhancement of antitumor activity and reduction of intestinal toxicity of irinotecan by the Chinese herbal medicine PHY906 (KD018)," BMC Complementary and Alternative Medicine, vol. 14, no. 1, p. 490, 2014.

[39] Y. Qiu, J. Zhang, R. Ji et al., "Preventative effects of seleniumenriched Bifidobacterium longum on irinotecan-induced small intestinal mucositis in mice," Benef Microbes, vol. 10, no. 5, pp. 569-577, 2019.

[40] R. Kon, Y. Tsubota, M. Minami et al., "CPT-11-induced delayed diarrhea develops via reduced aquaporin-3 expression in the colon," International Journal of Molecular Sciences, vol. 19, no. 1, p. 170, 2018.
[41] Z.-Z. Fang, D. Zhang, Y.-F. Cao et al., "Irinotecan (CPT-11)induced elevation of bile acids potentiates suppression of IL10 expression," Toxicology and Applied Pharmacology, vol. 291, pp. 21-27, 2016.

[42] Q. Li, X. Zhang, W. Wang et al., "CPT-11 activates NLRP3 inflammasome through JNK and NF- $\kappa$ B signalings," Toxicology and Applied Pharmacology, vol. 289, no. 2, pp. 133-141, 2015.

[43] Z. Zhang, L. Cheng, J. Li et al., "Inhibition of the wnt/betacatenin pathway overcomes resistance to enzalutamide in castration-resistant prostate cancer," Cancer Research, vol. 78, no. 12, pp. 3147-3162, 2018. 